\title{
Transfer of Critically III COVID-19 Patients in Low- Resource Setting Hospitals: Are We Doing It Right?
}

\author{
Shraya Banerjee ${ }^{1} \quad$ Nidhi Gupta $^{1}$ \\ ${ }^{1}$ Department of Neuroanaesthesia and Critical Care, Indraprastha \\ Apollo Hospital, New Delhi, India
}

J Neuroanaesthesiol Crit Care:2020;7:148-149

\section{Introduction}

The COVID-19 pandemic in the year 2019-2020 has left us questioning the practices we follow for transferring critically ill patients from the ICU to radiological suites or any other step-up or stepdown facility. In our settings with limited resources, although separate areas for COVID-19 confirmed, suspected, and non-COVID-19 areas have been identified, a mix-up of patients is often encountered due to lack of space and trained staff. Moreover, with no separate corridor or lift for COVID-19 patient transport and a shared common route of movement inside hospital, there is high-risk of disease transmission among healthcare workers and bystanders. Here, we share our experience from a 718-bedded tertiary care hospital in India with 32 dedicated COVID-19 ICU beds.

\section{Challenges Faced while Transporting ICU Patients: Practical Considerations}

Transportation of patients who are critically ill and on ventilator is a challenge for the accompanying anesthesiologist or any other healthcare support staff because of the risk of rapid deterioration. ${ }^{1}$ Needless to mention the stress of waiting time and unavailability of emergency response team immediately if patient condition declines. The issues are magnified because of limited experienced manpower and lack of clear standard operating procedures to be followed for COVID-19 patient transportation. The fear of contracting virus among the supporting staff makes it more difficult to get any radiological, diagnostic, or therapeutic procedures done in COVID-19 patients in places remote from the ICU.

The primary problem lies in generation of high-load of aerosols during bag and mask ventilation with a Bain's breathing circuit or an air mask bag unit (AMBU), ${ }^{2}$ the two most used devices for ventilating the patient lung during transportation. Aerosols can contaminate the trajectory of patient transport, apart from exposing the healthcare workers, security personnel and fellow passengers in the lift

DOI https://doi.org/ 10.1055/s-0040-1714184 ISSN 2348-0548.
Address for correspondence Shraya Banerjee, MBBS, MD, Department of Neuroanaesthesia and Critical Care, Indraprastha Apollo Hospital, Sarita Vihar, New Delhi 110076, India (e-mail: drshreyalhmc@gmail.com).

without proper protection. Moreover, the CT scan/MRI suite gets infected and poses risk of virus transmission in other susceptible individuals. In countries like India, where designated radiological suites for CT/MRI of COVID-19 patients are not available, this problem hits hard.

Beyond issues during transportation, the anesthesiologist faces unique challenges in radiological suites, with frequent disconnections of the ventilatory circuit from the endotracheal tubes, leading to aerosolization during patient shifting to $\mathrm{CT} /$ MRI machines; problems in assessing the requirement of appropriate sedation or patient counselling because of protective gear which makes communication difficult. Cardiopulmonary resuscitation in the event of cardiac or respiratory failure in the radiological suite or during patient transport can be challenging due to the heavy protective gear which makes hearing and vision unclear, reflexes slow, and communication problems between resuscitation team members. Fear of exposing oneself to heavy load of aerosols adds to the difficulty. ${ }^{3}$

\section{Possible Solutions to Address COVID-19 Patient Transport-related Issues in Limited Resources Setting}

Based on our working experience, we think the following measures and precautions are possible within limited resources settings to address the above-mentioned challenges ( - Fig.1).

Training and retraining of all the healthcare workers (doctors, nurses, housekeeping staff, security personnel, radiographers) involved in patient transport of suspected or confirmed COVID-19 patients on infection prevention and control (IPC) should be the first step. ${ }^{4}$ Available audiovisual tools can be used to educate on virus transmission and precautions to be taken to avoid transmission such as donning and doffing of personal protective equipments (PPE), hand and respiratory hygiene, safe handling of equipment, and contact precautions. ${ }^{5}$ All healthcare staff should understand their specific roles according to job profiles and act as a team to deal with any untoward event during patient transportation. Practice 


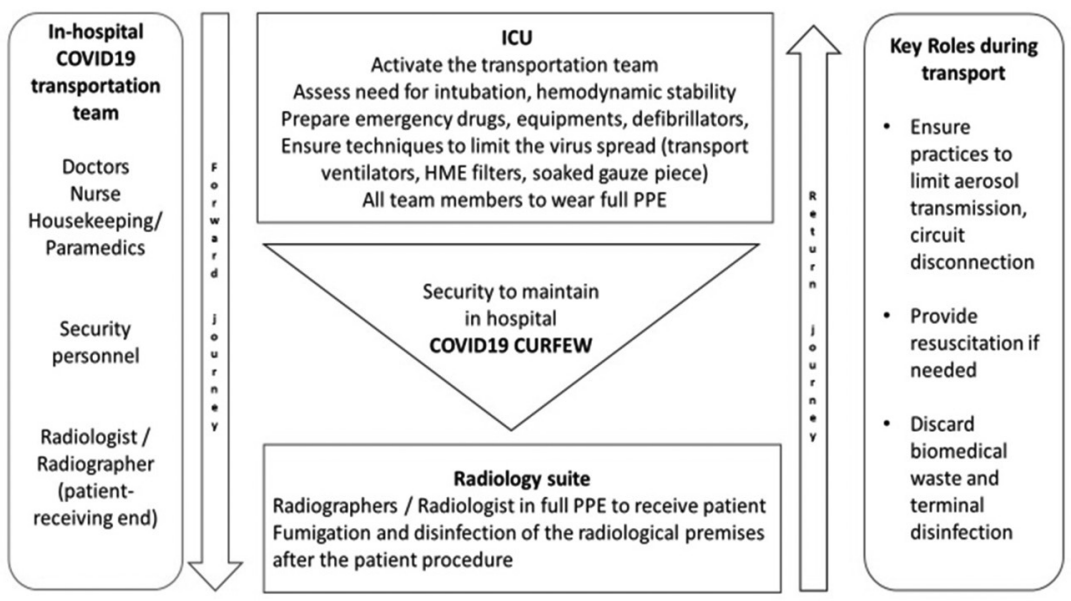

Fig. 1 Schematic diagram of in-hospital transportation of COVID-19 patients from ICU to radiology suite.

sessions on simulators for donning and doffing of PPE, transportation, resuscitation, and intubation can help to allay anxiety and fear of dealing with COVID-19 patients.

The major problem of aerosol generation during bag and mask ventilation can be decreased with closed circuit transport ventilators attached with heat moisture exchange (HME) filter., ${ }^{6,7}$ These filters are light-weight and small and have an efficiency rate of above $99 \%$ to trap bacteria and viruses. However, given its high-cost, in limited resources settings, one simple technique of wrapping the adjustable pressure limiting (APL) valve of the Bain's circuit or AMBU with a wet gauze piece (normal saline or hypochlorite solution) can trap the aerosols successfully. Limiting the number of circuit disconnections and, if required, disconnecting the circuit from the point after the attachment of HME filter, using ET tube clamps in sedated-paralyzed patients, and zeroing the oxygen flow before disconnection can significantly decrease viral load in the environment. Possibly, appropriately sized transparent box, plastic hoods or C-arm covers can be used to cover the patient trolley, especially the head-end, during transportation to minimize aerosol spread.

Bedside diagnostic procedures like portable X-rays and ultrasound machines can avoid transportation. If the need for transportation to CT/MRI suite arises, the patient should be reassessed for intubation. If necessary, intubation should be done in the isolation room under controlled conditions by an experienced physician before transportation. This is to avoid emergency intubation and unnecessary exposure to high-load of aerosols during in-hospital patient transfer. In patients with hemodynamic instability and on high-ionotropic support, careful assessment to ascertain need for transportation is crucial. It may be worthwhile to allocate specific hours for necessary procedures, which need to be followed during COVID-19 patient transportation, so that the radiological premises and passageway can be fumigated and shut for stipulated time. Alerting security guards along the way to the radiological suite during onward and return journey, to keep clear off the hospital visitors and staff beforehand, can limit unnecessary exposure-in-hospital "COVID-19 Curfew." Designated COVID19 transportation-team, corridors, and lifts can significantly reduce the exposure rates. Fumigation of the radiological suite and disinfecting the lift and hospital premises after transportation of COVID-19 patient can prevent cross-contamination. Designated areas to remove the PPE after the completion of all procedures is necessary. Continuous monitoring of ECG, noninvasive blood pressure (NIBP), and oxygen saturation is essential along with preparing emergency drugs and defibrillator. Anticipating emergency situations and adequate precautions can overcome the risk of contaminating the hospital premises.

Continuous training, anticipation and preparedness, following stringent precautions along with predefining specific roles, can significantly reduce the human-to-human airborne transmission during transporting critical-stage COVID-19 patients while improving work performance. Protecting the existing healthcare staff from acquiring infection at work is central to counter the overwhelming threat of COVID-19 pandemic to the Indian healthcare system.

\section{Conflict of Interest}

None declared.

\section{References}

1 Parmentier-Decrucq E, Poissy J, Favory R, et al. Adverse events during intrahospital transport of critically ill patients: incidence and risk factors. Ann Intensive Care 2013;3(1):10

2 Chan MTV, Chow BK, Lo T, et al. Exhaled air dispersion during bag-mask ventilation and sputum suctioning-Implications for infection control. Sci Rep 2018;8(1):198

3 Wong JEL, Leo YS, Tan CC. COVID-19 in Singapore-current experience: critical global issues that require attention and action. JAMA 2020. Doi: 10.1001/jama.2020.2467

4 Wax RS, Christian MD. Practical recommendations for critical care and anesthesiology teams caring for novel coronavirus (2019-nCoV) patients. Can J Anaesth 2020;67(5):568-576

5 NCDC, National Guidelines for Infection Prevention and Control in Healthcare Facilities. New Delhi: MOHFW; 2020

6 Respiratory care committee of Chinese Thoracic Society. [Expert consensus on preventing nosocomial transmission during respiratory care for critically ill patients infected by 2019 novel coronavirus pneumonia]. Zhonghua Jie He Hu Xi Za Zhi 2020;17(0):E020

7 Wilkes AR. Heat and moisture exchangers and breathing system filters: their use in anaesthesia and intensive care. Part 1 - history, principles and efficiency. Anaesthesia 2011;66(1):31-39 